\title{
Laboreal
}

Volume 11 N² | 2015

Varia

\section{La alianza Teoría /Práctica en la obra del sociólogo del trabajo Georges Friedmann (1922-1977)}

\section{Catherine Teiger}

\section{Q OpenEdition}

\section{Journals}

Edición electrónica

URL: http://journals.openedition.org/laboreal/3810

DOI: 10.4000/laboreal.3810

ISSN: 1646-5237

Editor

Universidade do Porto

\section{Referencia electrónica}

Catherine Teiger, « La alianza Teoría /Práctica en la obra del sociólogo del trabajo Georges Friedmann (1922-1977) », Laboreal [En línea], Volume 11 №2 | 2015, Publicado el 01 diciembre 2015, consultado el 24 septiembre 2020. URL : http://journals.openedition.org/laboreal/3810 ; DOI : https://doi.org/ 10.4000/laboreal.3810

\section{Este documento fue generado automáticamente el 24 septiembre 2020.}

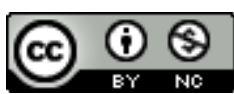

Laboreal está licenciado com uma Licença Creative Commons - Atribuição-NãoComercial 4.0 Internacional. 


\title{
La alianza Teoría /Práctica en la obra del sociólogo del trabajo Georges Friedmann (1922-1977)
}

\author{
Catherine Teiger
}

\section{REFERENCIA}

Comentario del texto: Friedmann, G. (1954). Intervention au XIème Congrès International de Psychotechnique (Psychologie appliquée), Section de psychologie du travail, Paris, 1953. Le Travail Humain, 17, 1-2, 39-40.

\section{NOTA DEL EDITOR}

http://dx.doi.org/10.15667/laborealxi0215ct

Para que este comentario no exceda el tamaño (¡demasiado!) del texto de Georges Friedmann (1902-1977), propuesto en este número de Laboreal, me limitaré a situarlo en su contexto histórico personal, disciplinar y social, y a referirme a una de las preguntas de fondo que planteaba a sus contemporáneos. Pregunta epistemológica que aún se sigue planteando aunque los contextos hayan cambiado. De esta forma, me inscribo dentro de la reflexión del sociólogo belga Mateo Alaluf (2001, p.102, traducción libre): "El regreso a los debates que se les podría pensar antiguos sigue siendo el mejor antídoto para preservarnos de la amnesia que nos acecha sin cesar en las ciencias sociales y fragiliza tanto nuestros resultados". Es por esto que los debates que han marcado la post guerra periodo de grandes transformaciones, que es también el del surgimiento de la ergonomía y de una nueva sociología del trabajo en los años 50- siguen siendo de tanta actualidad.

Este texto -sin otro título que el indicado aquí- proveniente de las Actes du XIème Congrès international de Psychotechnique (Psychologie appliquée ${ }^{1}$ ) - Sección de Psicología del 
Trabajo que tuvo lugar en Paris en 1953. Es el resumen de una reacción del "filosofopsicólogo-sociólogo" Profesor Georges Friedmann (1902-1977) a las ponencias de la sesión plenaria inaugural. Su intervención se sitúa después de las de los ingleses C. B. Frisby (1954)² ("Alocución inaugural") y L. S. Hearnshaw (1954) ("El objetivo y área de la psicología industrial"), de la del francés Pierre Goguelin (1954) ("Los factores psicológicos susceptibles de aumentar la productividad de las empresas") y de la del sueco Gunnar Westerlund (1954) ("Marco de referencias y problemas relacionados en psicología industrial”). Dicha reacción está más directamente enfocada en la intervención de L. S. Hearnshaw, es decir sobre la definición del área de la psicología del trabajo

Georges Friedmann (G.F.) evoca al respecto, entre otras cosas, las relaciones entre teoría y práctica, apoyándose en una declaración anterior (1932) de Jean-Maurice Lahy ${ }^{6}$ (1872-1943) a la que luego me referiré. Sin mencionar directamente a P. Goguelin, pero estando de acuerdo con la psicóloga Suzanne Pacaud, habiendo sido colaboradora de J.M. Lahy por mucho tiempo, G.F. menciona los riesgos de fracaso de una "política de la productividad" que no tomase en cuenta las interrelaciones entre todas las dimensiones del trabajo y que no mereciera así la "colaboración de psicólogos industriales que no queriendo comprometer su actividad poniéndola al servicio de intereses particulares sino, más bien, mantenerla en un campo de estudio científico". Esta política de la productividad estaba en pleno auge después de la guerra bajo la impulsión del plan Marshall de los EE.UU., plan de ayuda a la reconstrucción y la modernización de los países de Europa devastados por la guerra, y bajo la influencia de las "misiones de productividad" que entonces visitaron los EE.UU. Estas abarcaron todos los medios profesionales ${ }^{7}$ (Kuisel, 1988) a pesar de la oposición de los comunistas que denunciaban un "proyecto de catequesis de la clase obrera" (Brucy, 2001, p.81, traducción libre). Recordemos que una de esas misiones en 1956 -"El proyecto" o "Adaptación del trabajo al ser humano"- contribuyó al surgimiento de la ergonomía en Europa (AEP, 1959; Teiger \& Lacomblez, 2013). Se encuentran también rastros del descubrimiento de una precedente "Misión psicotécnica" (en 1952 con los psicólogos entre los cuales S. Pacaud et Jean-Marie Faverge) por ejemplo, en los capítulos del L'analyse du travail. Facteur d'économie humaine et de productivité (Ombredane \& Faverge, 1955) (Teiger, 2015a).

\section{¿Pero quién era Georges Friedmann (1902-1977)? Flashes bio-bibliográficos ${ }^{8}$}

Todo el mundo está de acuerdo en describir a G.F. como una de las cabezas emblemáticas de esos intelectuales progresistas de la post segunda guerra mundial (1939-45), seducidos entonces por el ideal comunista de justicia social y "compañeros de ruta" del Partido Comunista Francés (PCF), hasta la decepción y la ruptura final en los años cincuenta. Pionero de una "sociología humanista", contribuyó de manera decisiva a la nueva sociología francesa y en todo a la promoción de la sociología del trabajo. Comprometió toda su vida dedicándola a las evoluciones del trabajo humano en relación con las evoluciones del mundo técnico y evolucionando el mismo en sus análisis en función de las realidades que observaba.

Nacido en 1902, ingresa a la Ecole Normale Supérieure de Paris (ENS) en donde pasa su concurso de filosofía. Posteriormente, gracias al crédito aportado por la Fundación 
Rockefeller a la ENS (Tournès, 2008), trabaja como asistente durante 3 años, de 1932 a 1935, en el Centro de documentación social, ubicado en la ENS ${ }^{9}$ como docente asistente para los estudiantes de la Escuela. Con el fin de comprender mejor el trabajo obrero, sigue a tiempo parcial en 1931-1932 un curso de ajustador en la escuela profesional Denis Diderot en Paris, pero nunca trabajará como obrero en una fábrica.

Viaja a URSS tres veces (en 1932, 1933 y 1936) y usara esas observaciones (Friedmann, 1934) para su reflexión sobre el maquinismo en su tesis publicada en 1946: Problèmes humains du machinisme industriel (Friedmann, 1946), introduciendo así en Francia la nueva sociología del trabajo. Viaja también a los EE.UU.

Enseña después de 1935 a 1939 en la escuela profesional Boulle, de donde lo excluyen al aplicar las leyes antijudías del gobierno de Vichy vigente durante la ocupación de Francia por la Alemania nazi. Se enrola en el movimiento de Resistencia en la región de Toulouse, y vive en la clandestinidad. Sin embargo, el 23 de junio de 1941 tiene lugar una "Journée interdisciplinaire de psychologie et d'histoire du travail et des techniques", propulsada por el psicólogo Ignace Meyerson (1988-1983) y organizada por la Société d'études psychologiques de Toulouse, tras su creación en mayo de 1941. Las Actas -"El trabajo y la técnica"- no fueron publicadas hasta 1948; se les registra en términos elogiosos en la revista "Annales" por el historiador Lucien Febvre (1951), habiendo él mismo participado a esa Jornada ${ }^{10}$. Entre las ponencias figura el articulo importante de G.F. que L. Febvre presenta como "nuestro amigo": "Esquisse d'une psycho-sociologie du travail à la chaîne". Es la primera teorización de los "Problemas humanos del maquinismo industrial" (tema del trabajo de su tesis publicada en 1946) en donde afirma que el trabajo en la línea de montaje representa "una etapa histórica del desarrollo de la sociedad industrial" y que "los problemas del trabajo humano son a la vez problemas técnicos, problemas psicológicos y problemas sociales" (Friedmann, 1941-48, p. 127, traducción libre). L. Febvre concluye así su revisión crítica: "Se ve el interés de este número (de la revista "Annales") muy exitoso. Esta recopilación honra a aquellos que lo intentaron y el número será útil y seguirá vivo por mucho tiempo" (1951, p.243, traducción libre).

Después de la guerra, G.F. es nombrado Inspector general de enseñanza técnica (en 1945), profesor de Historia del trabajo en el Conservatoire National des Arts et Métiers (CNAM, de 1946 a 1960), Director de estudios en la École Pratique des Hautes Etudes (EPHE, de 1949 a 1960), Director del Centre d'Etudes Sociologiques (CES) del Centre National de la Recherche Scientifique (CNRS) de 1949 a 1954). Funda en 1959 con otros la revista Sociologie du travail. Finalmente, a principios de los años 60, explora otra área de la cultura técnica: las comunicaciones y la cultura de masa.

En 1962, G.F. coordinara con Pierre Naville el importante Traité de sociologie du travail ${ }^{11}$ (Friedmann \& Naville, 1962) ${ }^{12}$ después de la publicación en 1958 del Traité de sociologie coordinado por Georges Gurvitch, en el cual había escrito con Jean-Daniel Reynaud « Sociologie des techniques de production et du travail ». En 1953-54, época de su intervención en el congreso que nos interesa aquí, G.F. es una autoridad en el área de las ciencias del trabajo. Reorientó las actividades del CES que hasta ese entonces organizaba sobre todo conferencias. Con él, el CES se convirtió en un laboratorio de investigaciones. Una nueva "formula de investigación" se había puesto en obra con las entrevistas ${ }^{13}$ y las observaciones (como en EE.UU), aunque la práctica de investigaciones colectivas existía en Francia desde los años 30, época en cual surgió una política de las ciencias sociales ${ }^{14}$ (Tournès, 2008). 
El contexto de los años 50 facilita la evolución de esa sociología gracias a las oportunidades que aportó la creación en 1951 -bajo el impulso del ministerio del trabajo para la formación de los consejeros del trabajo- del Instituto de ciencias sociales del trabajo (ISST) perteneciente a la Universidad de Paris. Su sección de investigación se creó en 1954 con Yves Delamotte ${ }^{15}$, secretario general del nuevo Instituto del cual G.F. se vuelve el director. El jurista Marcel David ${ }^{16}$ tomará su lugar en 1959. Este último había creado, a fuerza de coraje, en la misma época (en 1955), el primer Instituto de trabajo en la facultad de derecho de la Universidad de Estrasburgo, para la formación superior de los sindicalistas nombrados para negociar en los órganos paritarios que se acababan crear (David, 1982).

Para alimentar esas formaciones de nivel superior con un objetivo concreto no académico, fue necesario realizar investigaciones empíricas actualizadas. Lo que reforzó una tendencia emergente en la época de la post guerra. Habían sido realizadas grandes investigaciones en la industria por G.F. en el CES a partir de 1949: las de A. Touraine y Etienne Verey en las plantas Renault (Touraine, 1955), las de Viviane Isambert-Jamati en la industria relojera (1955) y las de Maurice Verry en las laminadoras en las Ardenas (1955). Tales estudios fueron facilitados por los créditos importantes aportados por el plan Marshall a partir de 1954 al ISST entre otros ${ }^{17}$. Por lo tanto, las investigaciones que G.F. inició entonces sobre "las actitudes de los obreros de la siderurgia ante los cambios técnicos" se inscriben efectivamente en proyectos apoyados por el plan Marshall (en el marco de la Organización Europea para la Cooperación Económica/OECE), realizados de manera conjunta en Alemania, Bélgica, Francia, Gran Bretaña e Italia.

En lo que se refiere a Francia se trata de la investigación sobre los obreros de las siderurgias que se llevó a cabo en Mont-Saint-Martin (1954-1957 con J.-D. Reynaud, A. Touraine y sus colaboradores) y la de los funcionarios de los Cheques postales parisinos (Crozier, 1956). En el marco de la investigación en la siderúrgica de Mont-Saint-Martin, los estudios de campo fueron realizados por investigadores jóvenes ${ }^{18}$ que se pusieron ellos mismos el nombre de los "Gastón". G. Rot y F. Vatin (2008) publicaron y analizaron el diario de a bordo de los Gastón. Ilustrado con dibujos humorísticos, refleja los asombros, los descubrimientos, las dificultades de su labor, así como las reacciones de los investigadores novatos ante la división del trabajo intelectual que ellos estaban viviendo: ¡ellos en el campo día y noche, mientras que los jefes en sus oficinas en Paris esperaban los reportes!

Esto nos lleva a la pregunta de fondo planteada en el texto de G.F., que es la de la relación entre teoría/ práctica, ciencias fundamentales/ ciencias aplicadas.

\section{Teoría / Práctica; laboratorio / campo. ¿Porqué, dónde y cómo hacer ciencia y qué tipo de ciencia?}

La problemática central de G.F. que retendré en el marco de esta reseña es la relación entre teoría y práctica (o teoría y empirismo) pues, en la actualidad atraviesa en la actualidad a la mayoría de las ciencias humanas y sociales que estudian al trabajo humano como ser la ergonomía, la psicología, la sociología o la filosofía. Este mismo cuestionamiento se puede encontrar del lado de los epistemólogos - historiadores de las ciencias tales como la etología (Despret, 2011). 


\section{Teoría y práctica: ¿De qué se trata?}

Es conocido el adagio humorístico atribuido al físico Albert Einstein, padre de la teoría de la relatividad: "La teoría, es cuando se sabe todo pero nada funciona. La práctica, es cuando todo funciona y nadie sabe por qué. Acá, hemos reunido teoría y práctica: jnada funciona y nadie sabe por qué!".

La intervención de G.F., titular desde hace ya 7 años de la cátedra de "Historia del trabajo y relaciones industriales» del CNAM, en el XIavo Congreso Internacional de Psicotécnica es la ocasión para sentar su punto de vista respecto del enfoque del trabajo. Relacionado con el desarrollado de la consideración del Factor Humano, G.F. pone de manifiesto los límites de una concepción que separe a la teoría de la práctica. Evoca la posición J.-M. Lahy (1932) que sostenía que la psicología aplicada, o psicotécnica, no es otra cosa que la psicología científica general. Defendía que, no solamente no difiere de la psicología teórica sino que, nacida de ella, la sobrepasa, la transforma y la reemplazará como el producto de una evolución necesaria, como una síntesis de la teoría con la nueva práctica. Quizás se podría concluir que ¡Todo funcione y no se sepa porqué! En la misma lógica, 70 años después, el antropólogo Maurice Godelier $^{19}$ (2000, p.15, traducción libre), sostiene que: « las investigaciones en ciencias sociales pertenecen desde el principio a la esfera de la investigación básica (...) Son, como las ciencias duras, ciencias fundamentales que pueden tener aplicaciones, pues no existe verdaderamente una ciencia aplicada sino aplicaciones de la investigación».

Este tema recubre al menos cinco aspectos que se encuentran relacionados :1) el de los lugares y métodos privilegiados de producción de conocimientos, 2) el del valor de los conocimientos producidos, de acuerdo al lugar de producción, 3) el de la utilidad social, 4) el de la formalización y su difusión, 5) el de la evolución de las teorías. No hago más que mencionar los puntos que siempre están a la orden del día, al menos en ergonomía. Estos puntos son el objeto de Jornadas de estudios en dónde las cuestiones surgidas de la práctica plantean cuestiones teóricas que interrogan a las disciplinas y contribuyen a su desarrollo (cf. por ejemplo, Les Journées annuelles de l'université de Bordeaux sobre la práctica de la ergonomía, el Seminario transversal del l'Institut National d'Etudes sur le Travail et l'Orientation Professionnelle - Groupe de Recherches sur l'Histoire du Travail et de l'Orientation à Paris, en 2013, sobre las relaciones estudio de campo / laboratorio, sobre la intervención, etc.).

\section{(1) Los lugares y métodos privilegiados de producción de conocimientos: dos acepciones de las relaciones teoría / empirismo, campo / laboratorio}

Si la legitimidad y la investigación de campo está actualmente fuera de duda, quedan varios puntos por solucionar. Realizar estudios de campo pero ¿Para hacer qué cosa?

Examinando los argumentos desarrollados para legitimar el lugar acordado a los datos empíricos en el camino intelectual de producción de conocimientos sobre el trabajo en particular (Teiger, 2015b), se constata que un enfoque de campo lleva a dos argumentaciones contrastadas. Por un lado, la idea del estudio de campo subordinada a la teoría: como una extensión del laboratorio, el campo serviría para confirmar o refutar las hipótesis que la teoría había ya establecido a priori, aportando un valor suplementario de "validez ecológica ", sin preocuparse de los problemas de los actores 
del campo. Por otro lado, la idea del estudio de campo abordado con una gran apertura a los propósitos y a la actividad real de los actores, a los temas que espontáneamente aportan, sin el encuadre de las teorías estructuradas a priori, pero con una preocupación por contribuir a la resolución de cuestiones que los actores del campo se plantean.

Siempre en el campo de la sociología, y oponiendo estos dos enfoques de estudio de campo, se captura mejor el carácter innovador de los sociólogos friedmanianos. En Francia, hasta 1954, y las primeras grandes investigaciones de campo solicitadas por el Estado, la primera posición dominante en el ámbito de los métodos era la «sociología filosófico ${ }^{20}$ / teórica ». Pero, a partir de este período los Friedmanianos inventan realmente la nueva « sociología empírica » con, según M. Alaluf (2012, p.727, traducción libre) "la preocupación metodológica (que) ocupará un lugar central, como prueba de objetividad, en la elaboración de esta nueva sociología». Pero, según Olgierd Kuty (2008, p.58, traducción libre), "este empirismo era denigrado. La sociología afronta la indiferencia, y también la hostilidad Citando a Johan Heilbron (1991 , p.366-377, traducción libre), este autor concluye: «Entre los polos opuestos al "compromiso " y de "la pericia" (...), Les era extremadamente difícil encontrar una vía propia donde todavía la sociología se encontraba en una especie de no man's land, paralizado por la angustia de ser demasiado político (y por lo tanto no lo bastante intelectual) y, al mismo tiempo, de no ser demasiado político, no estar suficientemente al servicio de la sociedad ».

Se encontrarán reacciones cercanas a esta, por ejemplo cuando los ergónomos salieron de los laboratorios, diez años más tarde, en los años sesenta, para comprender mejor el trabajo y contribuir a actuar sobre él, a partir de « la demanda social » de la época (es decir los efectos poco visibles del trabajo en las líneas de montaje sobre la salud de los obreros y obreras en la producción de masa y en el transporte público). Con un triple efecto que Alain Wisner (1985) puso en relieve: la necesidad de inventar nuevos métodos para abordar las situaciones reales con una metodología científica deliberadamente asociada a la acción, la evolución conceptual que se desprende y transforma la definición y el objetivo mismo de la ergonomía y, finalmente, el desprecio e, incluso, la hostilidad de los partidarios del método experimental tradicional y de la pretendida neutralidad de la ciencia (Teiger, 2007, 2015b).

En ergonomía y en psicología del trabajo, a título de ejemplos de tal metodología, debemos citar el advenimiento de investigaciones-intervenciones denominadas participativas en Francia hacia fines de los años sesenta y, en Italia, en los años setenta ${ }^{21}$ el emplazamiento de la «comunidad ampliada de investigación» bien formalizada por el equipo de Ivar Oddone y sus sucesores intelectuales (Oddone et al., 1977; Muniz, et al., 2013; Lacomblez et al., 2014).

\section{(2) El valor de los conocimientos}

Encontramos acá la cuestión epistemológica del valor jerarquizado de los conocimientos, de acuerdo a los métodos y su producción que, con mucho humor, la epistemólogo-etologista belga Vinciane Despret (2011) instala en el centro de la reflexión. Despret pone en evidencia, en ciertos casos, los conocimientos "prácticos " adquiridos por los cuidadores y domadores de animales, que son cordialmente despreciados por los investigadores del dominio permitiendo, en cambio, obtener 
resultados a los cuales las experiencias "científicas» de laboratorio no pueden alcanzar $^{22}$, por ejemplo, enseñarle a hablar a una grácula religiosa recalcitrante.

Pero, realizar estudios de campo no es suficiente: ¿A quién, para qué van a servir los resultados de la investigación?

\section{(3) La utilidad y la responsabilidad: « la iluminación y la deuda »}

Esto nos lleva a la cuestión ética de la utilidad de las investigaciones, de los conocimientos producidos y de sus destinatarios, así como la responsabilidad de los investigadores / profesionales con relación a los lugares de trabajo, y de aquellos que viven de los mismos.

¿A quiénes beneficiarán los conocimientos ${ }^{23}$ ? ¿A la carrera de los investigadores o la empresa y, dentro de ésta a quiénes en particular? O, aún, ¿A las instituciones? Es así que se ha visto que los friedmanianos se beneficiaron de cierto reconocimiento a partir de la mitad de los años cincuenta, al menos la de los poderes públicos que juzgan su trabajo como de utilidad y los financian.

«La iluminación y la deuda », esta expresión del sociólogo Michel Crozier (1922-2013), teórico del actor en el sistema, resume bien las dos caras de la relación con la investigación de campo y los actores con quienes los investigadores / profesionales trabajan (Kuty, 2008). Por un lado, iluminación por el sentimiento de comprender mejor la realidad del trabajo y, por otro lado, la deuda incluyendo ésta responsabilidad con relación a aquellos con quienes se trabaja y que se les debe «dar» algo de los beneficios de la comprensión, adquiridos gracias a ellos. Según M. Alaluf (2012, p.729, traducción libre), en la tradición de Friedamnn «los investigadores deberán ser profesionales de una ciencia útil, en oposición a la sociología general ». La teoría sociológica no es un fin en sí mismo. Debe ser útil, producir conocimientos prácticos que puedan ser una herramienta de cambio, permitiendo a los interesados comprender mejor las situación en la cual se encuentran y, por lo tanto, de estar mejor e incluso cambiarla. ¡Los ergónomos no pueden menos que adherir!

Nos vemos por lo tanto llevados a plantearnos la cuestión de los destinatarios de la utilidad de las investigaciones, cuestión sobre la cual siempre existen profundos desacuerdos, presentando posiciones extremas. Años más tarde, M. Godelier (2000, p. 15, traducción libre ) subraya la utilidad social de las ciencias sociales: « Aquellos que se comprometen (...) lo hacen creyendo que sus investigaciones van a ser útiles tanto para ellos como para los otros ». Y prosigue: « ¿Qué recubre ese deseo de ser útil? La cuestión es compleja y las « utilidades » son muy diferentes, de acuerdo a al tipo de disciplina ». Del otro lado, por el contrario, están quienes recusan cualquier noción de utilidad.

Es el caso, por ejemplo, según Gabrielle Varro y Anne-Sophie Perriaux (1991, p.11, traducción libre) las posiciones adoptadas por los sociólogos C. Durand y A. Touraine en los años dans les años setenta: " En el importante, pero poco difundido informe de Durand y Touraine (1970), se precisa que no es el rol de la investigación realizar propuestas y recomendaciones de orden gestionario, siendo su objetivo teórico y científico».

La cuestión se plantea de modo diferente para la ergonomía en Francia que, luego de su fase « experimental» hasta mediados de los años sesenta, muestra claramente desde entonces un doble objetivo de conocimiento y de acción sobre la situación de trabajo. 


\section{(4) Consecuencia ético-metodológica: formalización y difusión de los conocimientos}

Desde hace ya mucho tiempo, sostenemos que en ergonomía y en psicología del trabajo una fórmula de investigación-intervención que pone el acento en la co-elaboración de la investigación con los actores de campo involucrados, acompañándose así de una formación recíproca en análisis del trabajo a partir de los conocimientos y de los puntos de vista de cada uno. Cada uno sale enriquecido en su dominio, resultando posible una acción común. Sin embargo, una acción parece indispensable para la acción común en diferentes dominios, y es la formalización luego de la difusión en un formato más accesible de los conocimientos y de las diferentes herramientas de análisis desarrollados para la realización de las investigaciones- intervenciones (Teiger \& Lacomblez, 2013: cf. los informes sindicales en el DVD adjunto). Desde nuestro punto de vista, la reflexión sobre este aspecto forma parte de una operación realizada en la investigación de campo a fines de facilitar la apropiación, por parte de los interesados. Una de las formulas interesantes es la escritura en común (investigadores profesionales y los actores de campo involucrados) de un documento accesible a todos que podrá, luego, servir de objeto de debate y de herramienta de trabajo (Teiger \& Leal Ferreira, 2015). Pero, este tipo de publicación muy cronográfica no tiene ningún valor académico ni profesional; su realización está por lo tanto sometida a disposición y a la buena voluntad de los autores. Es insuficiente.

\section{(5) Una evolución teórica alimentada por los conocimientos surgidos de la práctica de la investigación de campo}

Un último efecto remarcable de G.F. de su contacto con la investigación de campo es el de la evolución de su teorización respecto del trabajo en la línea de montaje, su objeto de estudio. Forma emblemática de la racionalización del trabajo y etapa histórica del desarrollo de la sociedad industrial. El trabajo en la línea de montaje representa para él, en un primer momento, un «mal necesario " esperando los beneficios descontados de la automatización que será liberadora. Ahora bien, este punto de vista que es el aspecto más conocido del pensamiento G.F., va a evolucionar (Rot \& Vatin, 2004) al tomar contacto con los resultados empíricos de los estudios e investigaciones realizados, en diferentes terrenos, por sus colegas y discípulos, y en función de sus experiencias directas de las situaciones industriales en diferentes, y contrastados, contextos nacionales (Francia, URSS, USA).

Leyendo los sucesivos textos G.F., es sorprendente, en el inicio, su ambivalencia. En sus textos de 1941/1948 hay una admiración e, incluso, fascinación por la proeza técnica que representa el trabajo en línea de montaje, símbolo vivo del maquinismo y de la racionalización del trabajo; pero, al mismo tiempo decepción y rechazo por resta forma de explotación del ser humano a la cual no podía restar insensible. Al inicio, toma posición contra « el concierto de maldiciones y gemidos» que tiende a explicar la crisis del mundo moderno mediante el reino deshumanizante de la máquina: $G$.F. pretende mostrar el drama social puesto de manifiesto por la "gran crisis » no se debe a la técnica, sino al capitalismo. Luego, evolucionará introduciendo nuevos elementos técnicos, a partir sobre todo de los trabajos de A. Touraine ${ }^{24}$, enriqueciendo su análisis a medida que avanzaban sus propias «investigaciones» en Rusia, USA y en Francia 
(Alaluf, 2012). La publicación en 1962 de un importante artículo: "La grande aventure » es la ocasión para G.F. de confirmar este cambio teórico y de tomar a contra pie sus trabajos iniciales. Mientras que, antes, todo su análisis apuntaba a distinguir el «buen » maquinismo (socialista) del "malo» (capitalista), desarrolla ahora la tesis de la convergencia entre los sistemas capitalista y socialista, expresiones gemelas de la misma "civilización técnica ». Qué importa entonces el marco político cuando se está " clavado » en la línea: "Y cincuenta horas semanales de trabajo en una línea de ensamblado de motores en una fábrica de tractores o de automóviles son, en sí, más " atrayentes " en Gorki que en Detroit ?» (Rot \& Vatin, 2004 , nota 69, traducción libre).

Se puede constatar una evolución teórica de la misma naturaleza en la ergonomía, en la obra de A. Wisner que pasó, a mediados de los años sesenta, de una investigación de tipo experimental aportando datos a los actores de terreno, pero sin implicarse en su eventual aplicación, al estudio directo de campo. Sin embargo, esto era considerado como «literario » no digno de ser publicado en las revistas científicas. Ahora bien, A. Wisner descubre que "Estos dos primeros estudios llevados a cabo con los sindicalistas planteaban a nuestro laboratorio cuestiones totalmente novedosas: metodologías, ciencias necesarias para nuestro progreso y relaciones con la vida social. Se trataba de un verdadero cambio! (...) Pero "realizar investigación de campo no quiere decir renunciar a establecer hechos científicos nuevos. Para retomar las palabras de un psicólogo inglés Tom Singleton: «Me preocupo por la psicología fundamental, y es por eso que voy a los lugares de trabajo» (Wisner, 1985, p. 30, traducción libre).

¡Esta será la palabra final!

\section{BIBLIOGRAFÍA}

AEP - Agence Européenne de Productivité (1959). Adaptation du travail à l'Homme. Recherches américaines et européennes sur les conditions de travail dans l'industrie. Paris : OECE (Organisation Européenne de Coopération Economique) éd.

Alaluf, M. (2001). L'analyse du travail et son organisation. Formation et Emploi, 76, 99-103.

Alaluf, M. (2012). Sociologie, in A. Bevort, A. Jobert, M. Lallemand \& A. Mias (coord.) Dictionnaire du travail (pp. 727-733). Paris : PUF.

Brucy, G. (2001). La doctrine de la CGT sur la formation des adultes : entre pragmatisme et lutte de classes (1945-1955). Travail et Emploi, 86, 65-85.

Crozier, M. (1956). Petits fonctionnaires au travail. Paris : Editions du CNRS.

Crozier, M. (2002). Ma belle époque : mémoires [1], 1947-1969. Paris : Fayard.

David, M. (1982). Témoins de l'impossible. Militants du monde ouvrier à l'université. Paris : Editions ouvrières.

Despret, V. (2011). Que diraient les animaux, si ... on leur posait les bonnes questions? Paris : Les empêcheurs de tourner en rond / La Découverte (rééd. 2014 La Découverte / Poche). 
Faverge, J.M., Browaeys, R., \& Leplat, J. (1956). Enquête sur les programmes de formation existant au sein des entreprises. Bulletin du CERP (Centre d'Etudes et de Recherches Psychotechniques), 6, 3, 235-324.

Febvre, L. (1951). Travail, travailleurs (revue bibliographique). Annales, 6, 2, 241-242.

Friedmann, G. (1934). Problèmes du machinisme en URSS et dans les pays capitalistes, Paris : Editions Sociales internationales.

Friedmann, G. (1941/1948). Esquisse d'une psycho-sociologie du travail à la chaîne, in Ignace Meyerson et al. (1948) Le travail et les techniques (pp. 127-144). Paris : PUF.

Friedmann, G. (1946). Problèmes humains du machinisme industriel. Paris : Gallimard.

Friedmann, G. (1950). Où va le travail humain ? Paris : Gallimard.

Friedmann, G. (1954). Intervention au XIème Congrès International de Psychotechnique (Psychologie appliquée), Section de psychologie du travail, Paris, 1953. Le Travail Humain, 17, 1-2, 39-40.

Friedmann, G. (1962). La grande aventure. Arguments, $\mathrm{n}^{\circ}$ 27-28.

Friedmann, G., \& Naville, P. (coord.) (1962). Traité de sociologie du travail. Paris : A. Colin.

Friedmann, G., \& Reynaud, J.-D. (1958). Sociologie des techniques de production et du travail, in G. Gurvitch (coord.) Traité de sociologie, t. 1 (pp. 443-460), Paris : PUF.

Frisby, C. B. (1954). Allocution inaugurale. Le Travail Humain, XVII, 1-2.

Glaser, B.G., \& Strauss, A.L. (1967). The Discovery Of Grounded Theory ; Stratégies for Qualitative Research. Chicago : Aldine de Gruyter.

Godelier, M. (2000). Le métier de chercheur. Sciences de l'Homme et de la Société-CNRS, 58, 12-18.

Goguelin, P. (1954). Les facteurs psychologiques susceptibles d'accroître la productivité dans l'entreprise. Le Travail Humain, t. XVII, $\mathrm{n}^{\circ}$ 1-2, 12-33.

Hearnshaw, L. S. (1954). Le but et le domaine de la Psychologie industrielle. Le Travail Humain, XVII ${ }^{\circ}$ année, 1-2, 4-11.

Heilbron, J. (1991). Pionniers par défaut. Revue Française de Sociologie, XXXII/3, 365-379.

Isambert-Jamati, V. (1955). L'industrie horlogère dans la région de Besançon. Paris : PUF.

Kuisel, R. F. (1988). L'American way of life et les missions françaises de productivité. Vingtième Siècle. Revue d'histoire, 17, 17, 21-38.

Kuty, O. (2008). La naissance de la négociation (1933-1962). Sociologies [Online], Theory and research, Online since 28 October 2008, URL : http://sociologies.revues.org/2483

Lacomblez, M., Teiger, C. , \& Vasconcelos, R. (2014). A ergonomia e o "paradigma da formação dos atores": uma parceria formadora com os protagonistas do trabalho, in F. P. Bendassolli \& L. A. Soboll (Org.), Métodos de pesquisa e intervenção em psicologia do trabalho: clínicas do trabalho (pp. 159-183; capítulo 7). São Paulo : Ed. Atlas.

Lahy, J.-M. (1932). Les fondements scientifiques de la psychotechnique. Communication à la VII ${ }^{\mathrm{e}}$ Conférence de psychotechnique, Moscou (1931), in Gouarné, I. (2007). Les conditions d'émergence du philosoviétisme dans l'univers de la psychologie scientifique française de l'entre-deuxguerres. Cahiers d'histoire. Revue d'histoire critique, 102 | 2007 : Sciences et politique. DOSSIER : La VIIe Conférence de psychotechnique (Moscou, septembre 1931), 65-87. 
Muniz, H., Brito, J., Souza, K. R., Athayde, M., \& Lacomblez, M. (2013). Ivar Oddone e sua contribuição para o campo da Saúde do Trabalhador no Brasil. Revista Brasileira de Saúde Ocupacional, 38, 280-291.

Oddone, I., Re, A., \& Briante, G. (1977). Esperienza operaia, coscienza di classe e psicologia del lavoro, Turín: Einaudi. (Trad. francesa, 1981: Redécouvrir l'expérience ouvrière. Vers une autre psychologie du travail, París: Éditions sociales).

Ombredane, A., \& Faverge, J.-M. (1955). L'analyse du travail. Facteur d'économie humaine et de productivité. Paris : PUF.

ROT, G., \& VATIN, F. (2004). Les avatars du « travail à la CHAÎNE » dans l'œuvre de Georges Friedmann (1931-1966). Genèses 4/2004, 57, 23-40. URL : www.cairn.info/revue-geneses-2004-4page-23.htm.

Rot, G., \& Vatin, F. (2008). L'enquête des « Gaston » ou les sociologues au travail. Jacques Dofny et Bernard Mottez à la tôlerie du Mont-Saint-Martin en 1955. Actes de la recherche en sciences sociales, 5 (175), 62-81.

Russell, S. (2015). Non aux robots de combat., le Monde, 8 août 2015, p. 14.

Teiger, C. (2007). De l'irruption de l'intervention dans la recherche en ergonomie. Education permanente, 170/2007-1, 35-49 (dossier Intervention et savoirs).

Teiger, C. (2015a). Jean-Marie Faverge en Amérique (1952) - Préludes à L'analyse du travail (1955). In R. Ouvrier-Bonnaz \& A. Weill-Fassina (coord.) L'analyse du travail, ruptures et évolutions. Toulouse : Octarès éd. (Téléchargement gratuit sur www.octares.com).

Teiger, C. (2015b). El laboratório. ¿Dónde "hacer ciencia”?, 11 (1), 121-126. (http.//dx.doi.org. $15667 /$ laborealx10115ct)

Teiger, C., \& Lacomblez, M. (coord.) (2013). (Se) former pour transformer le travail - Dynamiques de constructions d'une analyse critique du travail. Québec (Canada-Qc,) PUL-Presses de l'Université Laval et Bruxelles : ETUI-European Trade Union Institut éds.

Teiger, C., \& Leal Ferreira, L. (2015). Escrever juntos um livro (ergonomistas e sindicalistas): uma ferramenta para a dinamîca pesquisa - formaçâo - açâo. In F. M. G. Vezzá, A. M. Iguti, M. A. Bussacos, M. C. Gonzaga, \& S. Donatelli (org.), Análises do trabalho: escritos escolhidos / Lêda Leal Ferreira (pp. 51-62). Belo Horizonte, Fabrefactum Ed. Ltda.

Touraine, A. (1955). L'évolution du travail ouvrier aux usines Renault. Paris : Presses du CNRS.

Tournès, L. (2008). La fondation Rockefeller et la construction d'une politique des sciences sociales en France (1918-1940). Annales. Histoire, Sciences Sociales, EHESS, 63 (6), 1371-1402. (halshs-00652586)

Varro, G., \& Perriaux, A.-S. (1991). Les sens d'une catégorisation : « Les O.S. immigrés ». Langage et société, 58, 5-36.

Verey, É., \& Touraine, A. (1949). Enquête française de sociologie industrielle. Cahiers internationaux de sociologie, 7, 118-119.

Verry, M. (1955). Les Laminoirs ardennais. Déclin d'une aristocratie professionnelle. Paris : PUF.

Westerlund, G. (1954). Cadre de références et problèmes liés en psychologie industrielle. Le Travail Humain, XVII ${ }^{\circ}$ année, 34-38.

Wisner, A. (1985). Quand voyagent les usines. Essai d'anthropologie. Paris : Syros. 


\section{NOTAS}

1. Los dos términos parecen ser utilizados de manera indiferente en aquella época, la Psicología del trabajo estaba bajo la Psicología aplicada / psicotécnica.

2. Director del National Institute of Industrial Psychology, Londres, presidente de sección.

3. Profesor de Psicología en la Universidad de Liverpool.

4. Profesor de Psicología del trabajo en el Conservatoire National des Arts et Métiers, Paris (de 1970 a 1989)

5. Profesor de Psicología social en la Stockholm School of Economics.

6. Nació en 1872 (falleció en 1943), investigador y practicante de la psicotécnica, J.-M. Lahy funda en 1924 el primer laboratorio de psicotécnica en la S.T.C.R.P. (Société de transport en commun de la région parisienne) y en 1931 el Servicio psicotécnico de los ferrocarriles del Norte. Director del laboratorio de Psicología aplicada de la École Pratique des Hautes Etudes en 1927, su maestro inicial, Edouard Toulouse, lo presenta entonces como "el más grande realizador de la psicotécnica científica". Es el co-fundador de la revista Le travail humain en 1933.

7. El articulo de P. Goguelin (1954) hace referencia a une encuesta sobre los participantes a 128 misiones (sobre 500 más o menos), en vías de definir el rol de la formación adecuada del Psicología industrial en une perspectiva de productividad.

8. Me inspire ampliamente de Mateo Alaluf (2012), Olgierd Kuty (2008), Gwenaëlle Rot \& François Vatin (2004)

9. El Centro de Documentación Social (centro financiado por el banquero y mecenas Albert Kahn y beneficiando de los fondos americanos de la Fundación Rockefeller), se fundó en 1920 por Célestin Bouglé (1870-1940), filósofo y sociólogo, profesor en la Sorbona en Paris, Director de la École Normale Supérieure a partir de 1935.

10. Entre los autores figuran I. Meyerson, fundador de la Psicología histórica, que en el prólogo define el trabajo como una conducta, el antropólogo Marcel Mauss (« Les techniques et la technologie ; plaidoyer pour cette dernière et essais de définitions »), el historiador Marc Bloch, (" Les transformations des techniques comme problème de psychologie collective »). La contribucion de L. Febvre (« Travail : évolution d'un mot et d'une idée ») es presentada por Fernand Braudel, historiador en la misma rúbrica de las Annales (1951, p. 243).

11. M. Alaluf (2012: 729) señala que L'analyse du travail. Facteur d'économie humaine et de productivité d'Ombredane \& Faverge (1955) es la obra la más citada en ese tratado de sociología del trabajo.

12. Traducido al español en 1963: Friedmann G. \& Naville P. (1963). Tratado de sociología del trabajo. México: Fondo de Cultura Económica.

13. De hecho la entrevista no tiene aún un lugar importante (Crozier, 2002).

14. Cf. Los estudios novadores en la época del Centro de Documentación Social del ENS con el apoyo de los fondos provenientes de la Fundación Rockefeller (Tournès, 2008).

15. Alto funcionario del Ministerio del Trabajo, Profesor de derecho social en el CNAM (de 1976 à 1991)

16. Profesor de historia del derecho en la Universidad de Estrasburgo.

17. En esta misma época otros centros de investigación como el Centre d'Etudes et de Recherches Psychotechniques (CERP) llevan a cabo investigaciones de campo sobre otros aspectos del trabajo tales como la de J.-M. Faverge, Renée Browaeys y Jacques Leplat en 1956 sobre los programas de formación profesional existiendo en las empresas.

18. Se trata de Jacques Dofny, Bernard Mottez y Adrien Morel, posteriormente de Claude Durand, Paul Pascon y Philippe Pigenet (Rot \& Vatin, 2008).

19. Ex-director del departamento de ciencias humanas y sociales del CNRS.

20. Recordemos que en Francia, hasta la autonomía académica, en 1947 para la psicología y en 1958 para la sociología, estas dos disciplinas formaban parte de la enseñanza de la filosofía. 
21. Período que, en sociología, corresponde a la defensa de método inductivo y cualitativo por Glaser \& Strauss (1967) que conduce una « teoría anclada en los hechos».

22. Ver los ejemplos en Laboratorio » (Teiger, 2015b) del Diccionario de Laboreal.

23. Cf. El artículo Stuart Russell en el diario Le Monde del 8 de agosto de 2015, p. 14: « No a los robots de combate» quien hace alusión a las reacciones « irresponsables » de ciertos científicos frente a las investigaciones que permitieron construir la bomba atómica.

24. Desde 1949 para Francia, Friedmann podía respaldarse en la investigación de. Verey y Touraine (1949) en la fábrica Renault de la que hace referencia en la edición de 1950 de Où va le travail humain? (Friedmann, 1950).

\section{AUTOR}

\section{CATHERINE TEIGER}

Groupe de recherche sur l'histoire du travail et de l'orientation (GRESHTO), Centre de Recherche sur le travail et le développement (CRTD), Conservatoire National des Arts et Métiers, 41 rue GayLussac, 70005 Paris, France

moufcat@gmail.com 